\title{
La scène, une île déserte
}

\section{André Green}

\section{(2) OpenEdition}

\section{Journals}

Édition électronique

URL : http://journals.openedition.org/shakespeare/1279

DOI : 10.4000/shakespeare. 1279

ISSN : 2271-6424

Éditeur

Société Française Shakespeare

Édition imprimée

Date de publication : 1 novembre 1994

Pagination : 167-191

Référence électronique

André Green, "La scène, une île déserte », Actes des congrès de la Société française Shakespeare [En ligne], 12 | 1994, mis en ligne le 01 janvier 2007, consulté le 01 mai 2019. URL : http:// journals.openedition.org/shakespeare/1279; DOI : 10.4000/shakespeare.1279 
COSMOPOLITISME ET INSULARITÉ 


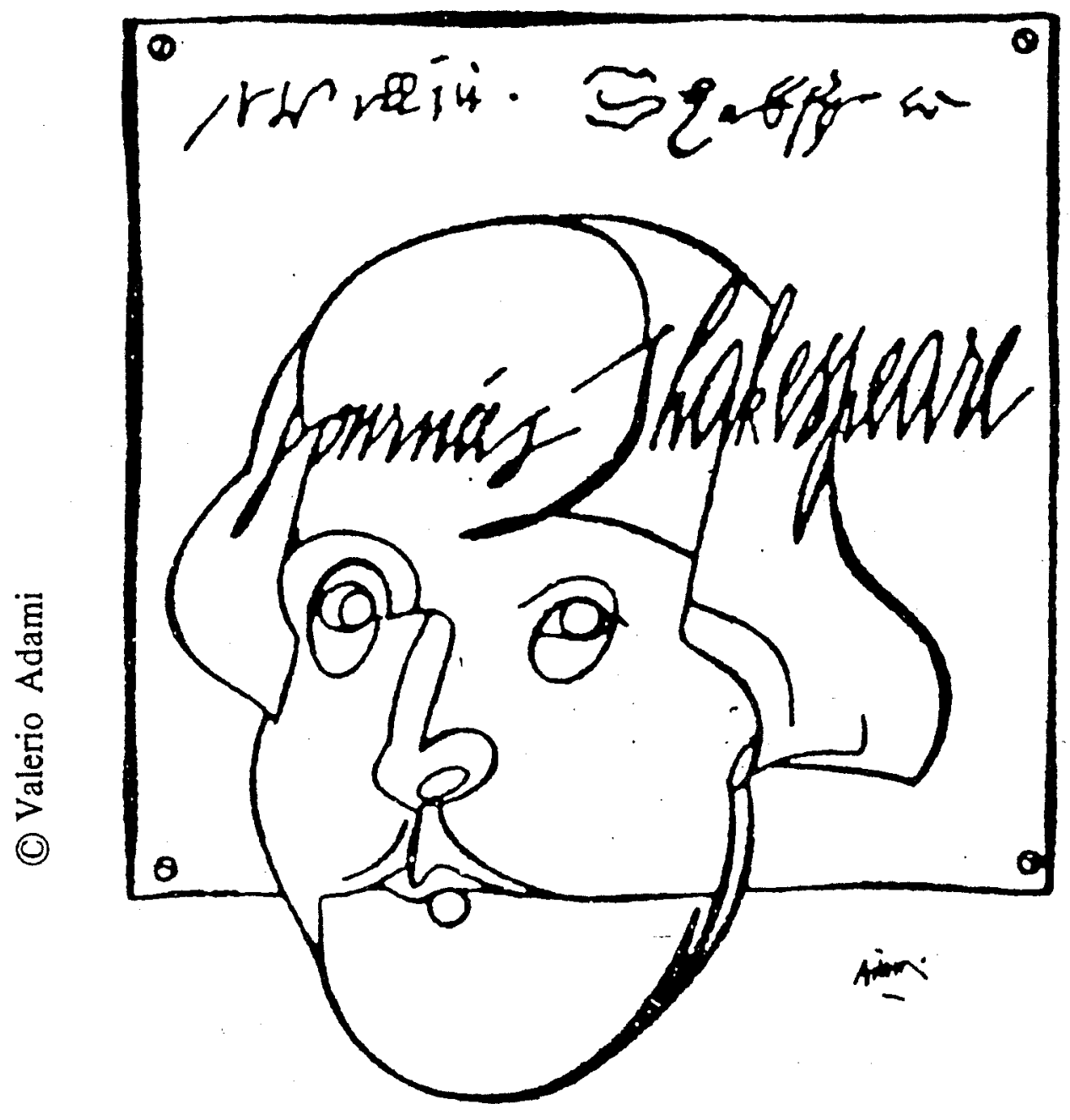

Affiche de Valerio Adami, spécialement conçue pour les journées Shakespeare 1979 


\section{SHAKESPEARE}

COSMOPOLITISME ET INSULARITÉ

Société Française Shakespeare

Actes du Congrès 1993

sous la direction

de

M. T. JONES-DAVIES

Ouvrage publié avec le soutien de

BARCLAYS

BARCLAYS BANK S.A

PARIS

LES BELLES LETTRES

1994 
Tous droits de traduction, de reproduction et d'adaptation réservés pour tous les pays.

(C) 1994 Société d'édition Les Belles Lettres, 95 bd Raspail 75006 Paris

ISBN 2.251.69122-7 


\section{La scène, une île déserte}

$\grave{a}$ Frank Kermode

Une des propriétés peut-être la plus forte - du chef d'œuvre est d'engendrer une infinité d'interprétations. Celles-ci sont multiples par le nombre des points de vue où elles se placent et parce qu'elles s'étendent dans la durée, chaque génération les abordant différemment. Conclusion: l'œuvre est une totalité intotalisable. Pas seulement ouverte : rebelle à toute insularisation. En outre lorsque celle-ci met un point final au travail d'un auteur prolifique, un bon laps de temps avant le terme de sa vie, elle n'est pas seulement le dernier maillon d'une chaîne mais constitue en ellemême une somme. Elle acquiert de ce fait un sens quelque peu différent de celui de son ouverture infinie si elle n'avait été qu'une parmi d'autres, le silence qui lui succède lui conférant, en tout état de cause, une valeur limitante définitive. Et quand enfin sa thématique explicite est celle de l'adieu et qu'elle se clôt sur une prière au public pour prendre congé, on atteint par là un concentré de données emboîtées les unes aux autres qui laissent augurer que non seulement l'interprétation ne pourra être simple ou univoque - ce qui est toujours le cas avec Shakespeare - mais qu'en l'occurrence les difficultés attendues en dévoileront bien d'autres.

En La Tempête se retrouvent - comme Wilson Knight n'a pas eu de peine à le montrer - des échos des romances précédentes ce qui permet de fructifier les expériences antérieures et surtout de porter à un haut degré d'achèvement le traitement de certains thèmes répétitifs déjà abordés que la relecture fait apparaître comme des ébauches en attente de leur solution. Effet, donc, de clôture après coup qui donne au mouvement de la création une fonction contenante ne se contentant pas de délimiter une identité mais faisant celle-ci dépositaire d'une très forte condensation. Cela pourrait expliquer la faveur que la pièce a connue et le nombre de questions qu'elle a soulevées. Ne faudrait-il pas faire preuve d'audace devant une constellation si singulière et l'éclairer à la lumière d'un mode de pensée qui devrait s'éloigner de la rationalité ordinaire, celle qui dans beaucoup de cas suffit à nous fournir des réponses qui, si elles 
sont loin d'épuiser le sujet, nous donnent néanmoins le sentiment d'une prise suffisante pour conduire l'investigation?

En effet, on ne peut manquer de remarquer que la conséquence de la tempête qui ouvre la pièce a été de dissocier ${ }^{1}$ en plusieurs fractions - chacune réunissant un groupe social homogène évoluant pour son propre compte et sans contact les uns avec les autres et avec Prospero (à l'exception de Ferdinand) - les personnes transportées par la nef. En fin de compte, voyageurs et résidents de l'île seront tous réunis, à nouveau, peu après, toutes ces péripéties ayant été soumises à la règle des trois unités, dont Shakespeare n'était pas un observant scrupuleux, mais à laquelle il avait autrefois sacrifié dans ses premières comédies. Mieux encore le temps de l'action regroupe les trois dimensions du passé évoqué, du présent dramatisé et de l'avenir annoncé.

Les comédies et les romances sont construites autour de situations se développant selon une dynamique propre qui les mène à leur terme. Inévitable, la fin n'est pas toujours le moment le plus heureux de la création du poète. Nécessaire sans doute, mais d'une réussite aléatoire et pas toujours convaincante. Dans La Tempête, cela est différent : on dirait que la conclusion de la pièce est sa véritable raison d'être. Cela se renforce par son aspect "testamentaire". Ce point final est unificateur dans l'intrigue, après coup, et l'allusion directe à la situation thêâtrale, au temps ultime précédant la dissolution de l'œuvre, s'applique à la vie de l'auteur autant qu'à la pièce. Jean-Jacques Mayoux fera observer que des romances de la fin, La Tempête est la seule à se lire à plusieurs niveaux ${ }^{2}$.

Je me suis autrefois demandé comment Shakespeare avait pu continuer d'écrire après Hamlet ${ }^{3}$. Sans doute ai-je pensé à l'analogie avec ce qui se produit dans l'expérience analytique où après une phase particulièrement féconde de l'analyse au cours de laquelle beaucoup de choses s'éclairent et prennent sens, fait suite une reprise de l'activité défensive. On voit alors apparaître le retour à un matériel d'importance plus secondaire, fruit d'un déplacement du conflit principal. Je sais maintenant qu'il en a été ainsi parce que Shakespeare allait ensuite partir pour le long voyage qui devait nous conduire au port de l'île de la Tempête. «Hamlet is a student and a scholar; and in this too, as in his surface (though not actual) ineffectuality and his revulsion from an evil society he forecasts the learned Prospero» dit Wilson-Knight ${ }^{4}$. Il y a loin cependant de l'un à l'autre et sans doute fallait-il que l'orage de King Lear errant sur la lande et trahi par les siens soit passé pour attendre que 
La Tempête ne permette de reéditer ce qu'un autre cataclysme avait accompli défaisant l'autorité paternelle et la raison du roi ${ }^{5}$. Tout le chemin qui va du fils en deuil de son père, juge implacable de la trahison de sa mère, à celui du père préparant sa sortie finale, imprécateur impitoyable de la trahison de ses filles. Le premier est dit fou, et le second ne se serait jamais connu lui-même. Prospero est leur double et leur contraire. Il les achève en les dépassant et se dépasse lui même en rentrant dans l'ombre.

On a beaucoup discuté pour savoir dans quel genre classer La Tempête, la dénomination de romance paraissant incomplète sinon inadéquate. Sans nous attarder sur ce débat, dont l'argumentation est fournie, je me contenterai de brèves réflexions. Comme d'autres l'on pensé avant moi, je ne crois pas que la pièce soit une allégorie, elle manque trop à la transparence démonstrative d'une symbolisation que la convention rend facilement reconnaissable et dont l'effet vise à une édification qui frôle l'ennui. Elle n'est pas non plus une simple comédie rehaussée par quelques ingrédients de plus haute noblesse. Dire d'elle que c'est une pastorale tragicomique n'emporte qu' à moitié la conviction car l'impression qui en résulte est trop banalisée. Forçant un peu du côté de l'emphase, Wilson Knight n'a pas craint de soutenir qu'elle était un miracle chrétien. Miracle, sans doute, Coleridge l'avait perçu, mais miracle humain, miracle, me permettrai-je de dire, temporel. Ceci pour s'opposer à la caractérisation spiritualiste de tradition, poussée à son point le plus haut par Wilson Knight. Comme si l'on craignait que, faute de le rappeler à ceux qui prennent grand plaisir en la compagnie de Shakespeare au théâtre ou dans le cabinet de lecture, ils risqueraient d'être expédiés sans ménagement en enfer le jour de leur mort.

Miracle en effet, par la grâce, l'exotisme, les prodiges, la poésie musicale et colorée qui concourent tous à la félicité finale et miracle temporel car toutes ces vertus sont au service du temps. Du temps qui souverainement avance et auquel même les pouvoirs de la magie sont en fin de compte subordonnés bien qu'ils paraissent en jouer à leur guise. Marche qui consent, pour autant, au plus prestigieux de ses magiciens à lui laisser le plaisir de se jouer de ceux sur lesquels il a jeté son dévolu en leur faisant croire qu'il en est le Maître. Cela ne dépasse qu'à peine le pouvoir dont croient jouir les grands de ce monde, qui n'en subissent pas moins sa loi. «Le temps est un enfant qui joue avec des pions. Royauté d'un enfant», disait Héraclite. Qui ne perçoit que Prospero est un enfant? 
Lear nous avait habitués à nous en montrer les tragiques caprices, Prospero, par sa sagesse finale, nous rendrait-il plus optimistes? Après avoir déchaîné les éléments comme par une déchirure de l'élément sur lequel s'écoule le temps abouchant un passé qu'on avait cru révolu à un avenir que rien ne laissait préfigurer, après avoir arrêté les horloges, fait dormir en plein jour et rêver les yeux ouverts ceux qui ne sont là que pour servir le jeu du Maître, c'est le Temps qui triomphe enfin et reprend sa course à une allure droite qui remet chacun à sa place. Or la marche du monde serait déréglée si l'univers entier était sujet à cette arythmie. Pour que celle-ci puisse néanmoins avoir lieu et que de ces procédés magiciens nous tirions la leçon, il faut isoler - voilà le mot - ces péripéties. Deux lieux en un s'y prêtent. La scène, une île déserte : "an un-inhabited" island ; un-in : "non en". Par la subtile et implicite désignation spatiale négative ("rien dedans") Shakespeare nous fait un clin d'oeil en nommant ce lieu "I land", le pays du sujet, traversé par le Temps, parce qu'il n'est de sujet qui ne se définisse par rapport à luí.

Cette île inhabitée (remarquez au passage l'oxymoron par inadvertance que représente le bilinguisme pour la circonstance quand on n'opte pas pour le pléonasme : non habitée ou habitée du dedans; en fait l'île est bien à tous égards habitée d'habitants d'avant et d'après sa colonisation) définit encore mieux la pièce à la tombée du rideau quand chacun se prépare à rentrer chez soi, qu'à son lever lorsque le navire échoue sur ses côtes. «It might be said that the title of the play should be The Island, for the storm occupies only the first scene of the play and the island pervades all of it»?

Dès ces premières réflexions nous sommes bien obligés de voir qu'il nous faudra renoncer dans notre commentaire à une causalité identitaire linéaire, réglée par une rationalité trop stricte pour contenir cette lecture à plusieurs étages qu'appelle le spectacle. Et ce n'est pas seulement parce qu'il comporte une bonne part de féérie, de mythe et occasionnellement d'allégorie qu'il nous faut nous montrer plus disposés à accepter un mixte de raison et de déraison, mais parce que la rationalité propre de l'œuvre est sa vraie raison, c'est-à-dire celle qui inclut dans son intimité la plus profonde l'ombre de sa déraison avec laquelle la raison oblige à composer si elle ne veut ni s'automutiler ni succomber sous ses assauts déchaînés : la tempête est là pour nous le rappeler. Les vents furieux peuvent aussi nourrir les instruments dont sortent les airs harmo- 
nieux et le génie d'Ariel au service de Prospero, charme et excite la pensée de son maître. Son esprit d'air et de feu attire à lui ceux qui sont nécessaires à l'accomplissement des desseins de celui qu'il sert. Les forces de Caliban sont aussi domestiquées par Ariel sous le regard attentif et ordonnateur de celui qui se prépare à changer la prospérité de sa solitude en souci pour sa postérité.

A la fin de mon étude sur Hamlet dont le sous-titre - je me permets de le rappeler - était "Une interprétation psychanalytique de la représentation", je proposai de créer un néologisme pour un nom qui fait défaut dans notre langue : celui de "représenteur"8. Je désignai ainsi ce qui au thêâtre remplirait la même fonction que le narrateur dans le roman. Or c'est là l'essence de ce qu'est Prospero qui ne se laisse confiner dans aucun des personnages auxquels il renvoie : auteur, metteur en scène, comédien. Encore est-il essentiel de remarquer que s'il est circonstanciellement un duc spolié, et un magicien, il est, avec une importance au moins égale, un père diligent.

Un mot en ce qui concerne la discussion sur l'aspect autobiographique de l'œuvre. Cette tonalité me semble indiscutable dans La Tempête. Je veux dire qu'elle est plus perceptible que dans d'autres œuvres et je précise qu'elle n'est ni à récuser entièrement partout ni à invoquer partout au même titre. Dans La Tempête, Shakespeare s'expose davantage comme il le fait parfois et on ne manque pas de s'en apercevoir - je pense à ce que Jean-Jacques Mayoux a écrit à propos de Hamlet. Quelquefois l'argument est plus difficile à soutenir parce que Shakespeare se cache sous un personnage secondaire de la pièce comme dans le cas de Faulconbridge dans Le Roi Jean. Toutefois, évitons la méprise : il ne s'agit jamais, à proprement parler d'autobiographie. Shakespeare n'a aucune intention de se confesser à nous. Il est même vraisemblable que s'il prenait à un auteur l'envie de monter une pièce qui s'appellerait "Ma vie", ce que peut toujours faire un romancier, il y a toutes chances pour qu'il courre droit à l'échec. Théâtralement, cela risque fort de ne jamais décoller et pis encore c'est la vision de la vie qui se trouverait aplatie d'être portée à la scène. Cela tient à ce que le théâtre n'est pas un lieu où l'on se raconte - c'est un lieu où l'on montre et cela transforme nécessairement la nature de ce qui est à montrer. C'est pourquoi la biographie d'un autre peut être adaptée au théâtre, voire être écrite pour lui. Parce que le théâtre fait venir à la vie, la vie montrée devient nécessairement autre. C'est ce à quoi nous fait assister La Tempête - où le 
thêâtre accomplit sa vraie nature de nous ouvrir les portes d'une vie rêvée?

Autobiographie : au théâtre, ce terme est impropre et si l'expression n'était pas pédante, il serait plus juste de parler d' "émergence subjective dans l'espace thêâtral". Qu'est-ce à dire? Que l'auteur s'étant emparé d'un thème, d'une intrigue et se portant au devant d'eux, il est saisi à son tour par le travail de l'œuvre qui l'entraîne à s'incorporer au traitement de la matière théâtrale. Qu'un va et vient puisse alors s'établir entre ses intentions créatrices et le jeu grâce auquel il pense pouvoir entrer et sortir à son gré dans l'univers qu'il crée, cela semble plausible. Mais au bout du compte si le souci de la maîtrise artistique reste sa principale préoccupation, il ne s'en suit pas régulièrement que le créateur reste toujours maître de cette implication subjective. Davantage, nous ne sommes pas loin de penser qu'un certain degré d'échappement au contrôle est ce qui donne à l'art la touche qui transforme la poésie immanente en grâce incomparable.

Donc, oui, La Tempête est autobiographique, et sans doute Shakespeare en savait-il quelque chose, mais au bout du compte j'en suis persuadé - beaucoup moins qu'il ne le pensait Aurait-il su tout ce que sa Tempête recelait de trésors - comme la grotte de Prospero - il n'aurait guère ressenti le besoin de nous le montrer en nous le présentant sur la scène.

Wilson Knight a eu de beaux accents pour nous parler de l'autobiographie artistique ${ }^{10}$. Son jugement est peu discutable, mais il reste prisonnier - sans doute à cause de la métaphysique qui est la sienne - de sa vision hiérarchique, contredite par l'examen de la pièce et même par l'interprétation à laquelle elle donne lieu chez son commentateur. Nous nous en rendrons mieux compte quand nous reviendrons en détail sur le problème.

Avant d'entrer dans ces mystères, il faut nous arrêter à la composition de l'œuvre. Frank Kermode souligne son exceptionnelle économie. Paradoxalement, on signale sa pauvreté relative en images et la rareté des métaphores. Mais on n'a pas de peine à reconnaître que c'est parce que la pièce est en elle-même une métaphore que peut-être son agencement - on sait que le texte, particulièrement fiable, en l'occurrence, comporte ici la division en actes et scènes - est encore mieux apte à révéler cette dimension métaphorique. Esquissons en quelques phases son processus.

Le premier acte est de position: la tempête (sc. 1) en est l'introduction. Le transport dans lîle sera l'occasion d'une exposi- 
tion sur l'histoire du couple initial : Prospero-Miranda. Viendront s'y joindre, sans se rencontrer, Ariel puis Caliban, créatures de Prospero, ses intermédiaires masculins. Enfin l'acte se clôt sur la rencontre Ferdinand (extrait du lot des naufragés par Ariel) - Miranda dont l'union formera le couple final de la pièce, son but ultime. La Tempête est l'histoire du mariage de Miranda.

Le débarquement dans l'île par lequel débute le deuxième acte montre la dissociation des passagers : l'équipage disparaît de la scène. (Les pilotes de la raison sont mis en congé.) On voit les cours de Milan et de Naples (le roi croyant son fils perdu), errer, séparées de leurs serviteurs. Aussitôt nous sommes témoins d'une nouvelle intrigue usurpatrice qui répète les circonstances du récit de l'acte précédent et nous reporte douze ans avant. Le complot se déroule sous nos yeux, mais cette fois la tentative est mise en échec. Ariel veille. A un autre bout de l'île, voilà la basse cour, pourraiton dire, un valet et un bouffon qui n'ont cure que de leur plaisir le plus primitif : le vin. Après la préfiguration de l'union sexuelle, la criminalité pour la possession du pouvoir, enfin l'ivresse orale. Caliban qui prend d'abord les comparses pour des esprits, leur fait allégeance. Les deux moitiés des passagers du navire convergeront, chacune de leur côté, pour se réunir à nouveau à la fin de la pièce vers l'antre de Prospero. La basse cour est l'image dégradée de celle de ses maîtres mais, au fond, elle est sa (dérisoire) vérité. L'acte établit implicitement le rapport entre l'intrigue principale qui concerne l'avenir de Miranda et l'intrigue secondaire, le règlement des comptes du crime passé, toujours prêt à se renouveler, et le dévoilement du vrai visage des nouveaux habitants de l'ile, nobles ou valets, avides, insatiables naïs et sots.

Le troisième acte se répartit entre les épreuves de Ferdinand, prétendant à la succession de Prospero auprès de sa fille, qui ont la vertu d'accroître l'amour de Miranda et les péripéties qui entourent le projet d'un complot fomenté par Caliban et les serviteurs des cours d'Italie pour mettre à mort Prospero et s'emparer de son royaume. Quant aux nobles, ils deviennent le jouet d'Ariel qui abuse leurs sens aiguisés par la faim en leur présentant des fantasmagories et des chimères. En somme, les serviteurs repus et avinés deviennent comme les courtisans, ambitieux, et les courtisans qui rêvent de pouvoir, tenaillés par la faim en sont réduits à ne penser qu'à leur ventre vide. Mais en tout état de cause, c'est la fin de Prospero qui est préfigurée sous des formes diverses. 
Le quatrième acte présente d'autres chimères sous une forme allégorique avec une visée morale : parfaire l'initiation de l'époux, successeur du père auprès de l'épouse. Les rêves éveillés sont aussi poétiques qu'édifiants. Bientôt les sublimes visions s'évanouissent et l'on voit revenir la basse cour ivre de vin et enflée d'illusion.

Enfin le dernier acte est la mise en scène de l'ultime représentation de Prospero triomphateur, aidé pour la dernière fois d'Ariel. Retrouvailles avec les cours de Naples et de Milan. Distribution des mérites et des blâmes. Ferdinand est rendu à son père et fiancé à Miranda. L'équipage rejoint la troupe : donc retour de l'espoir du retour. Ridicule du complot de Caliban et consommation de sa défaite. Epilogue : requête de Prospero pour sa libération de l'ile et son retour au foyer. Reconnaissance du pouvoir du public.

Un schéma de composition tripartite se dégage. Trois groupes peuvent être comptés au départ : celui de Prospero avec les siens, celui des passagers, et celui de l'équipage qui met les deux précédents en communication. Ultérieurement l'équipage disparaissant de notre vue, le groupe des passagers se scinde en deux : maîtres et serviteurs. Prospero est en butte à ses anciens ennemis et ses nouveaux ennemis du moment : celui qui s'apparente à ses ennemis du passé deviendra son parent allié, Ferdinand. Une fille, princesse de son état, a été spoliée ; elle sera largement dédommagée en devenant la femme d'un futur roi. En chaque sous-groupe on peut remarquer des dédoublements. Ce que l'on appelle par abréviation la "Cour" se divise en fait en deux factions : celle des ennemis directs - le frère usurpateur - et celle des ennemis indirects - son suzerain napolitain. Autre division tripartite entre ceux qui sacrifient aux tentations du pouvoir et usurpent les titres de la légitimité apparente, ceux qui aspirent à s'emparer des titres précédents et ceux un peu benêts qui sont fidèles aux régimes qui se succèdent sans se mêler de prendre parti, Gonzalo, Adrien, Francesco. Il n'est pas jusqu'à cette version grotesque du complot, dont le but parait être de ridiculiser toute manœuvre du genre, qui n'obéisse à cette composition tripartite. Elle réunit un sommelier ridicule et un bouffon tous deux séduits par Caliban. Cette insistance de la structure ternaire doit peut-être être comprise par rapport à la finalité de la pièce : le rétablissement d'un ordre légitime comme promesse d'un bonheur à venir, devant passer par la triangulation fondamentale au cour de la pièce, celle des rapports Prospero-Miranda-Ferdinand. Personnage central, Prospero - dépossédé autrefois et menacé de même aujourd'hui encore — nous invite à nous moquer ré- 
trospectivement de l'usurpation dont il était la victime. La révolution de palais d'hier ne vaut guère mieux que l'invraisemblable et misérable conspiration des valets d'aujourd'hui. Une autre dépossession est à l'ordre du jour pour Prospéro, autrement douloureuse parce que légitime et naturelle, sa fille chérie étant en âge de prendre époux. Inexorable action du Temps que nul complot n'a besoin d'aider dans l'accomplissement de ses buts. Cela, qui n'est pas explicitement énoncé dans l'exposition, ajoute par son non-dit à la charge de mystère de la pièce. Nous comprendrons plus tard que c'est ce qui décide Prospero à revenir au monde et à quitter sa parure de magicien. Enfin, au plus profond du personnage, une dernière tripartition, celle qui associe le mage à ses deux serviteurs : Ariel et Caliban. Leur opposition a peu de signification si on ne la rapporte pas à ce qui fait leur lien : leur sujétion à un Maître commun. La mère n'est jamais nommée, Prospero occupant toujours la scène directement ou par émissaires interposés. Toutefois, il doit, avant de donner sa fille, veiller à son édification : c'est la fonction du masque qui fera descendre des cieux trois déesses Iris, Cérès, Junon. C'est ici que l'allégorie sert, à point nommé, à désigner l'absente au moment du mariage : la mère. Les deux dernières évoquent des associations suffisamment précises pour n'y pas revenir - encore qu'il ne soit pas superflu de rappeler au passage la condition de Proserpine, prisonnière d'Hadès - Iris, on le sait, est la messagère des Dieux, une sorte de double d'Hermès - dans ce contexte, nous dirons plutôt d'Ariel. Elle est une divinité associée aux puissances marines et servante de Zeus et d'Héra. Elle symbolise surtout la liaison entre la terre et le ciel (l'Arc en ciel). "Parfois, on en fait la femme de Zéphir et la mère d'Eros." 11

Cette trinité féminine qui réunit les liens du mariage (pas toujours facile), la condition maternelle (souvent tourmentée) et la "dédication" de la femme est bien de Shakespeare. Voilà l'une des données essentielles de cette insistance tripartite : un trio révèle autour d'un thème principal deux valeurs complémentaires ou antagonistes.

Ayant eu à cœur de reconnaître un aspect structural fondamental de la pièce, celui qui habite de l'intérieur cette île dite inhabitée, voilà que nous avions failli oublier le véhicule même de l'intrigue, qui est comme l'écrin de ces péripéties et qui, surprise! car aucune nécessité ne l'explique, est elle-même tripartite, l'équipage : "The Master, the Boatswain and the Mariners", dit la liste 
des personnages, qui deviendra dans la chanson à boire de Stephano "the master, the swabber, the boatswain and I" 12 (II,2,47), vers superbes aux accents qui font penser à Lewis Carroll. Ces gens de mer ouvrent la pièce, s'évanouissent et ne réapparaissent qu'à son dénouement. Au cas où nous douterions un moment du sceau de cette trinité répétitive, écoutons le bref propos du Boatswain annonçant la bonne nouvelle de la réunion de l'équipage avec leur Roi. Il ajoute encore ceci :

"the next, our ship

Which, but three glasses ${ }^{13}$ since we gave out split.

Is tight and yare and bravely rigged, as when

We first put out to sea."

$(\mathrm{V}, 1,223-225)$

La règle des trois unités est plus que respectée, la pièce se déroule sous le double signe du temps réel et des mystères magiques, mais le but est le même: revenir au rassemblement d'avant la catastrophe, et au delà encore au temps où Prospero régnait pacifiquement sur Milan.

Je crains qu'on n'ait pas pris garde à tout le poids symbolique dont Shakespeare a chargé cette ouverture de la pièce avec cette nef des fous ballotée sur la mer, dans la double dimension de l'épreuve présente, la Tempête, et des réminiscences qu'elle fait lever. Tout est dit en huit vers : le captaine - Master of the Ship - interpellant son Maître d'équipage, le Boatswain (un "swain" est un jeune berger, un amoureux de pastorale et plus généralement un soupirant), celui-ci répond à son appel.

"Here master : what cheer ?"

Il ordonne alors :

"Good : speaks to th' mariners : fall to it, yarely, or

We run ourselves aground : bestir, bestir" (I,1,2-4)

Le maître d'équipage s'exécute :

"High, my hearts! cheerly, cheerly, my hearts"

Un instant après il exigera des passagers de demeurer en bas - dans la cabine : chacun à sa place, afin que tous soient à leur poste.

Jamais plus le capitaine n'aura droit à la parole. 
Nous ne sommes pas ici dans l'allégorie mais dans la parabole. Ce Master of the ship, il est normal qu'il soit secondé, pas au point cependant de déléguer l'essentiel de sa fonction : commander en personne le navire dans la tempête. Cette entrée en matière condamne Prospero abandonnant le gouvernement de l'Etat et déléguant son commandement à un second. Qu'on se demène, qu'on s'active ("Fall to it, yarely"). Au dernier acte lorsque le navire est retrouvé intact, paré et gréé - light and yare - Prospero, le capitaine du navire, a enfin repris la situation en main. Parler à l'équipage, oui, mais s'adresser à son cœur. Presque en musique : my hearts, cheerly, cheerly, my hearts. Contre le vacarme des éléments le chant des âmes.

L'intrigue de La Tempête est construite sans modèle préexistant. Tout est de l'invention de Shakespeare qui aborde donc l'œuvre avec une totale liberté. Nous nous proposons d'aller du plus manifeste au plus latent et de là à l'idée de déterminants extratextuels dont la prise en considération ajouterait à l'intelligibilité de la pièce, sans méconnaître cependant leur nature hypothétique. La Tempête traite donc au premier abord du dénouement heureux d'un forfait commis à l'égard d'un duc versé dans la magie. Il en perd son trône, est exilé dans une île. Plus tard, les circonstances lui donnant l'occasion au moyen de l'art dans lequel il est versé, de tirer vengeance de ses ennemis. Il confond ceux-ci puis, renonçant à sa vindicte comme à sa magie, il rentre dans son duché après avoir marié sa fille au fils du suzerain de son traître de frère, le mariage rendant les deux duchés égaux.

Il est bien clair que cette réduction à quoi se résume la pièce ne dit rien de ce qu'elle est. Aller un peu plus au fond des choses nécessiterait que nous approfondissions le rapport Ariel et Caliban - ce dernier personnage ayant été perçu par la critique comme un des éléments les plus forts, sinon le plus fort de l'œuvre. Je ne vais pas plus loin pour le moment dans le traitement des intentions supposées de Shakespeare. Toutefois cette extension des pouvoirs de Prospero dans les deux directions que prennent les emplois de ses deux serviteurs nous conduit à identifier en fait trois thèmes traités par l'intrigue: le premier, initial, explicite, concerne l'ambition criminelle dont l'enjeu est le pouvoir qui ne triomphera qu'un temps, le deuxième, final, au profit duquel tout se résout, est l'amour qui va réunir les deux jeunes premiers dans le mariage, le troisième d'interprétation plus obscure mais qu'on pourrait désigner comme l'aspect secondaire du thème manifeste : 
le complot grotesque de ce que nous avons appelé la basse-cour. Ici l'ambition dérisoire est liée à l'ivresse. Arrêtons-nous sur ce troisième référent qui vient se placer à côté de l'ambition et de l'amour. On pourrait soutenir qu'en fait il n'y a complot qu'en apparence, Stephano consentant à tout ce que Caliban lui propose, pourvu qu'on lui fasse miroiter qu'il pourra satisfaire sans limite son désir de boire en la joyeuse compagnie de son alter ego, Trinculo. Aussi cette facette de la pièce joue-t- elle un rôle de contrepoids par rapport aux deux autres qu'elle imite en les rabaissant: l'ambition y est surtout l'expression de la vantardise, l'intérêt recherché celui de la panse et du cullit, selon le nom du bouffon. Le mauvais génie qui les inspire, Caliban, est une créature brute, prompte à donner licence à ses pulsions sans discrimination ni jugement. Non sans incohérence, il se propose de devenir l'esclave de ceux sur lesquels il veut s'appuyer pour se libérer de la tutelle d'un maître qu'il déteste. La Tempête ne serait pas ce qu'elle est sans cette face comique, de plus absolument nécessaire pour que puissent se déployer toute la palette des "qualités" de Caliban dont la paillardise, venant au premier plan, devient pitoyable. Tout comme l'imitation des grands en revêtant leur parure accentue encore l'écart de leur dissemblance avec eux. L'attachement de ces pitres à leur biberon devient encore plus lamentable lorsque ceux-ci parlant des bouteilles qui leur viennent à manquer usent de tournures nobles.

Trinculo: "Ay but to lose our bottles in the pool,

Stephano: There's not only disgrace and dishonour in that monster, but an infinite loss" (IV,1, 209-210)

Ne dirait-on pas qu'ils parlent de leur mère?

Seul un Caliban peut confondre le vin de table et le vin de messe. Lorsqu'il aperçoit pour la première fois le sommelier et le bouffon enivrés, il ne doute pas que cette condition soit en rapport avec la divinité.

These be fine things, and if they be not sprites

That's a brave god, and bears celestial liquor

I will kneel to him.

(II, 2,116-118)

Les esprits désignent dans la pièce des significations contradictoires. Le plus souvent ce sont des puissances domptées par Prospero pour servir ses desseins, donc ennemies de Caliban ${ }^{15}$. 
Que l'on songe aux punitions infligées par Ariel, quintessence de l'esprit de Prospero, aux deux larrons blasphémateurs : ils parodient le Livre en donnant la bouteille à baiser dans la tradition des farces comme s'il s'agissait de quelque symbole du sacré. Guidé par son maître Prospero, Ariel lâche sur eux des dogues qui se repaîtront de leur chair, appelés entre autres Argent, appât du pouvoir. Ce châtiment contraste avec le supplice raffiné qu'il réserve aux gens de la Cour. Affamés, il leur donne l'illusion qu'un riche banquet leur est offert, pour les décevoir cruellement aussitôt après, la table du festin s'évanouissant.

Que Shakespeare prenne un plaisir évident à écrire ces scènes ne veut pas dire qu'il se contente ici de s'amuser, quelle que soit sa familiarité avec les situations de ce genre. Car en les associant à Caliban, il nous pousse à rechercher une signification plus profonde cachée derrière leur trivialité. La symbolisation n'est pas l'apanage des situations réservées aux personnages nobles de la pièce. Pas besoin pour cela de rappeler que la relation de Dionysos à la sexualité n'est pas dissociable de son rapport à la vigne.

$\mathrm{A}$ un autre moment de la pièce, quand Antonio invite Sebastiano à se débarrasser d'Alonso, le poussant à un crime analogue au sien, quoique plus grave encore, il lui fait entrevoir le bonheur d'une souveraineté sans limites. Car pour ceux qui seront ses sujets :

They'll take suggestion as a cat laps milk;

They'll tell the clock to any business that

We say befits the hour.

(II, 1,282-284)

Pourtant si rabaissée que soit l'image de l'homme présentée par les comparses en lesquels Caliban voit ses sauveurs, nous savons que Shakespeare ne refuse pas de s'y reconnaître. Il admet qu'elle est une partie inaliénable de l'humanité. Il serait presque reconnaissant à ces pauvres diables de pourvoir encore à notre joie en dépit de leur misère psychologique. Si Caliban est le sauvage "déformé", l'homme de la nature, la caricature de ces cannibales décrits par Montaigne dont Shakespeare ne partage pas la vue optimiste, Trinculo et Stephano sont bien ses cousins éloignés. Le peu de culture qu'ils ont acquis en tirant les ressources de la nature, ils n'en ont pas fait un usage si nettement meilleur que Caliban dont Prospero regrette le peu d'instruction qu'il lui a donné et qui n'a eu d'autre résultat que d'empirer son état. Tous les défauts qui lui sont reprochés, éclos à la suite de ce nuisible apprentissage, on 
peut les reconnaître chez Trinculo ou Stephano, au désir de viol de Miranda près, avec la vanité en plus. Mais l'épisode comique du monstre à quatre jambes, n'est-il pas une aimable galéjade faisant allusion à la bête à deux dos ? Que l'un des deux soit un "bouffon" nous apprend qu'il peuvent aussi faire rire d'eux-mêmes et pas seulement de leurs maîtres. Qu'importe, l'essentiel est de ne pas laisser la gravité prendre toute la place et de ne jamais oublier les "cheers", même pour saluer les illusions les plus enfantines. Nous aurions ici un paradigme du dicton célèbre selon lequel la faim et l'amour mènent le monde. L'ivresse fait le pont entre les deux.

Frank Kermode pense que La Tempête traite essentiellement des rapports entre la Nature et la Civilisation - en fait il dit "Education", dont le sens dépasse de beaucoup l'homonyme français, on le sait.

Il règne dans La Tempête, une atmosphère sonore singulière. Sa gamme est riche et étendue. Elle va de l'inquiétante étrangeté des bruits qui peuplent une île déserte, présages de menaces surgissant de partout, aux airs mélodieux qui charment l'oreille d'une harmonieuse musique dans une paix tranquille propice à la jouissance sans souci. Ainsi se succèdent le rugissement de la furie maritime, le fracas du navire qui se brise en morceaux, les sonorités insolites de l'environnement attribués à la présence de monstres, les airs naturels plus doux vantés par Caliban :

the isle is full of noises

Sounds and sweet airs, that give delight and hurt not

Sometime a thousand twangling instruments Will hum about mine ears : and sometime voices

(IV, 2, 131-116)

Les voix humaines entonnent les refrains, parfois pour égayer les humeurs avinées, parfois pour donner aux charmes d'Ariel l'enchantement des airs gracieux, accompagnant parfois les danses des silhouettes féériques. Ici agit l'art de Prospero. Le spectacle lyrique culmine dans le Masque, prélude aux noces à venir du dénouement heureux. L'intrigue de La Tempête est économique, sa composition rigoureuse mais la fluctuation de son évolution entre des genres divers nécessite qu'un fil relie les styles, les genres, les types humains et par dessus tout rappelle l'omniprésence de Prospero. Ce fil c'est la musique encore plus que le langage, ou mieux encore le noeud de l'entrelacement de leurs fils. Plus précisément 
encore, la musique accentue les contrastes et retient l'ensemble sous les auspices de cet au-delà du langage devant lequel le poète le plus inspiré sait s'incliner.

Venons en à Caliban. 11 faudrait toujours avoir en tête la chanson par laquelle il se désigne lui-même : "Ban-Ban Caliban", comme un enfant qui s'amuse. La critique a reconnu dans ce personnage une création puissante et non une simple commodité dramatique (Frank Kermode). 11 est surprenant que Shakespeare le fasse s'exprimer en vers. S'il est une représentation de ces hommes découverts dans les îles et les continents du Nouveau Monde, il n'en est pas le fidèle portrait. Cela fait 19 ans que Colomb les a décrits et Shakespeare n'était pas homme à s'en désintéresser. L'image est ici délibérément métaphorisée - un sauvage "déformé" dit la distribution. Déformé par quoi ? Si on ne veut pas s'arrêter à l'explication qui fait de lui le rejeton d'une sorcière et du diable, Caliban est-il déformé par l'esclavage et les mauvais traitements de Prospero ou par la vision que nous en avons, nous servant de sa différence d'avec nous pour en faire le réceptacle de nos projections? L'important est que Shakespeare qui n'a pas hésité à forcer le trait sur sa mauvaise nature, donne à son discours les accents de la poésie. Au point qu'un Wilson Knight n'hésitera pas à voir en lui un précurseur des héros nietzchéens. Jean-Jacques Mayoux ne va pas jusque là, mais reconnâît qu'il y a en lui du poète.

Je crois que l'audace de Shakespeare va beaucoup plus loin : il ose proclamer que la veine poétique, dans ses effets les plus divers, prend sa source dans cette part la plus primitive de notre nature bien qu'on tente de la domestiquer comme si elle n'était que nuisible. Part la plus insoumise et la plus obstinée à demander qu'on l'entende, quoiqu'aussi la plus portée à servir les maîtres les moins aptes à cette fonction pourvu que ceux-ci nourrissent nos illusions. La civilisation pense avoir fait disparaître le Caliban d'autrefois, elle ne le reconnaît pas sous les traits de ce qui perdure de lui en ceux qu'elle a civilisés. On nomme cela la pulsion. Shakespeare, en avance sur Freud, annonce de façon voilée à ses contemporains que même la grâce d'Ariel a retenu quelque chose qui viendrait de Caliban. L'art ne supplante pas les traces de cet homme primordial, ni ne se contente de l'habiller de ses atours, il tire son énergie de lui : l'amour le nimbe d'une brume qui laisse apparaître la silhouette des amants occupés à des jeux édifiants, sage exercice de l'intellect fasciné, derrière les rideaux du fond de la 
scène. Quant au pouvoir, s'il a décuplé sa puissance par les ruses de l'intelligence et les détours de l'industrie, il sait aussi se forger les raisons qui rendent encore plus opaques ses origines et le goût qu'il suscite à toutes les époques.

Voilà même qu'il nous est montré que lorsque l' "education" réussit à surmonter la résistance obstinée au changement chez celui qu'on appelle le primitif, la domestication des forces qui animent Caliban prendra la forme de la compulsion à répéter : Antonio après avoir spolié Prospero veut obtenir de Sébastien qu'il fasse de même avec Alonso. Pour sauver toute cette gent de son insatiable appétit, il faudra les touchantes utopies de Gonzalo. En somme, quand ce n'est pas la perfidie qui renverse les obstacles à la satisfaction de ses appétits, le jugement succombe sous les coups de l'illusion. Quel est l'intérêt pour le développement de l'intrigue de cette tentative d'assassinat collatéral ? Il faut une meilleure explication que le simple désir de mettre sous nos yeux la déloyauté que peut dicter l'ambition. De toute manière, Prospero - dès lors que ses ennemis sont entre ses mains - n'en fera qu'une bouchée. De si grands moyens ne peuvent se justifier par une visée seulement négative : le châtiment des coupables. 11 leur faut un but plus élevé. L'omniprésence déguisée de Caliban chez ceux qui semblent lui ressembler le moins.

En vérité, tous les personnages empruntent quelque chose à la nature de Caliban. Parfois, cela est d'emblée reconnaissable selon le proverbe, qui se ressemble s'assemble - chez Stephano et Trinculo, mais aussi, à travers leur intermédiaire, à l'équipage du navire ivre selon une tradition établie. Nous en avons des échos plus déguisés chez Antonio et Sebastien criminels accomplis ou potentiels. Mais oublierions-nous la complicité d'Alonso à la forfaiture de l'actuel duc de Milan ? Et Gonzalo, tout honnête qu'il soit, n'est-il pas demeuré pour servir l'usurpateur? Même Ariel, pourtant entièrement dévoué à la cause de son maître, est traité par lui de "malignant thing", fut-ce pour rire (I, 2, 257). Shakespeare traque les désirs mauvais de l'homme sauvage jusque dans les pensées les plus apparemment innocentes des âmes nobles. Ainsi Prospero est-il prompt à chapitrer Ferdinand lorsque celui-ci Shakespeare a deià traité la situation de manière inoubliable, pensons au prince Hal d'Henri IV - paraît un peu pressé de s'asseoir sur le trône de Naples qu'il promet à Miranda ${ }^{17}$. 
thou dost here usurp

The name thou ows't not

et l'accuse de vouloir le dépasser aussi.

and hast put thyself

Upon the island as a spy to win it

From me, the lord on't

(I, 2, 456-459)

On croirait entendre parler du fils de Sycorax, s'il avait eu la chance d'aller à l'Université. A l'inverse de ce qu'on pourrait attendre, Prospero n'est guère idéalisé. Il a des pouvoirs magiques mais sa nature d'homme, avec toutes ses faiblesses n'est pas difficile à reconnaître. Peut-être n'atteint-il à la sagesse du renoncement, à la vengeance vis à vis de ses ennemis, que parce qu'il est parvenu à consentir à un sacrifice plus grand encore : donner sa fille à un autre. Il réussit là où Lear avait échoué. Il s'est aussi résigné à se séparer d'Ariel qui est loin d'être un ange et ne cache guère son plaisir à se jouer - sur ordre de son maître - des ennemis de Prospero.

Un seul personnage échappe totalement à ce que représente Caliban : la parfaite Miranda, mais pourquoi ? Je me risquerai à une réponse : parce qu'elle a été soustraite à l'influence de sa mère - sans d'ailleurs que nous sachions rien d'elle sauf ceci :

Thy mother was a piece of virtue

She said thou wast my daughter; and thy

Father was Duke of Milano.

Etrange manière de présenter une femme de si haute vertu ! La mère de Prospero n'est pas traitée de manière moins ambiguë par Miranda qui réagit au récit de la trahison de son oncle.

I should sin

To think but nobly of my grand-mother

Good wombs have bom bad sons

Si donc Miranda est sans tache, c'est à son père qu'elle le doit. Et s'il est un parfait éducateur, c'est grâce à sa science aidée de magie. Donc une mère disparue dont la fille aura même oublié le visage (III, 1,49), remplacée par un père jouant les deux rôles, pour le plus grand bien de l'enfant.

D'où l'importance de ce service d'esclavage de Ferdinand qui, se justifie par sa valeur initiatique et éducative. Celle-ci est d'autant plus nécessaire quand on est le fils d'un souverain à la morale 
un peu lâche, le roi de Naples, qui marie sa fille au roi de Tunis, peu soucieux du grand écart qui sépare les mondes où les époux ont grandi ${ }^{18}$. Ferdinand devra donc reparcourir, sous la tutelle de Prospero, l'itinéraire humain qui, parti d'une condition proche de Caliban - il est affecté au même service que lui - le fera parvenir jusqu'à celle de parfait époux, partenaire et adversaire au grand jeu d'échecs de l'amour, ne trichant que pour faire gagner celle qu'il aime. N. Holland suit le fil de ce bois naturel et mythique ${ }^{19}$. Depuis celui dont les arbres sont faits qui emprisonne les êtres comme en un ventre maternel, à celui qui sert à fabriquer les pièces des jeux d'échecs ${ }^{20}$. Or il fait remarquer que bois dans la langue de l'époque signifie bestial. On le voit, Shakespeare ne se range pas à quelque vision bêtifiante du rapport entre époux : il les montre jouant à la guerre, sans la faire, chacun ayant plus à coeur la victoire de l'autre que la sienne. Ils vécurent heureux ... Fin traditionnelle de romance ou de tragicomédie pastorale, certes. Ultime tentative de gagner les faveurs des spectateurs dont on prendra congé tout à l'heure ? Sans doute, mais authentique pensée shakespearienne et sens dernier de cette spiritualité qui jamais ne peut oublier les fondements matériels et charnels de l'amour. Il ne se réclame, en fait, que conventionnellement de la religion ${ }^{21}$.

La critique a trop négligé cette fonction d'éducateur aux rites de l'amour de Prospero : le masque, ce spectacle somptueux est à l'usage exclusif des futurs époux. Consacré à l'apologie de l'affection féminine, il est aussi le couronnement de l'apprentisage de Ferdinand. On ne comprend guère pourquoi Ariel, sur ordre de Prospero, doit prendre l'apparence d'une nymphe marine au début de la pièce, mais c'est à l'évidence pour séduire Ferdinand dont on sait qu'il prise le commerce des femmes. Il jurera plus tard à Miranda qu'elle ne saurait se comparer aux autres qu'il a connues. La sirène attirera Ferdinand sur des voies qui l'éloigneront du reste des naufragés, c'est-à-dire de sa famille. Et quand il paraît subjugué à la vue de Miranda, je n'ai pas de doute quant à moi que ce coup de foudre aura bien bénéficié d'un coup de pouce venu de Prospero.

The Duke of Milan

And his more braver daughter could control thee If now 'twere fit to do't. At first sight

They have changed eyes.

$(\mathrm{I}, 2,442-445)$

Prospero montre à Ferdinand la dureté, il l'assujetit, allant même jusqu'à le traiter de Caliban en s'adressant à sa fille qui, elle, 
le prend pour un esprit. Ce faisant, il excite très efficacement la compassion de Miranda. Quand les jeunes gens se seront déclarés, il dira, ayant assisté incognito à leur échange :

So glad of this as they 1 cannot be,

nous serions surpris s'il en restait là, mais il ajoute pour notre confort :

Who are surprised with all

La levée de ce qui un instant nous aura inquiétés nous empêche peut-être de nous arrêter à une interprétation plausible : celle que Prospero aurait manigancé la naissance de l'amour entre eux.

Revenons un moment sur cette autobiographie spirituelle que serait cette Tempête selon Wilson Knight. Nous le ferons pour reprendre les éléments de son argumentation et les prolonger dans une direction autre. L'autobiographie spirituelle est à l'autobiographie artistique ce qu'est l'autobiographie symbolique (fantasmatique) à l'autobiographie événementielle. Il y a autant de mystère dans cette influence du symbolique sur les événements de la vie que dans celle de la spiritualité sur l'art. Sur quoi repose une telle élaboration symbolique? Sur ce que j'ai appelé les structures subjectives qui, lorsqu'elles interviennent sur ce plan concernent ce que Lacan appelait des signifiants clés : ceux de la filiation, de la paternité, de la différence des sexes et des générations etc.

11 n'est donc pas contingent de rappeler que Shakespeare voit et sa petite fille naître et sa mère mourir en 1608, deux ans au plus avant l'écriture de la pièce. Certains critiques pensent que Shakespeare s'était déjà retiré à Stratford avant d'écrire La Tempête. On peut supposer que le retour à son foyer aurait été en rapport avec ces ou cette mort(s). Cinq ans après La Tempête, disparaît son auteur. Quand Shakespeare écrit sa pièce, Judith est agée de 26 ans et est toujours célibataire ${ }^{22} .26$ ans, lâge où Anne Hathaway devait épouser Will, enceinte de ses œuvres.

Le chiffre douze a été relevé par la critique. La chute de Prospero à Milan a lieu douze ans avant le commencement de la pièce. $(1,2,53)$. Ariel est resté emprisonné pendant douze ans dans un tronc d'arbre par la volonté de Sycorax. (I,2,279). Wilson Knight se permet une excursion pour rappeler que le cycle des grandes pièces, tragiques, remonte douze ans avant, à peu près. Autrement significatif pour Shakespeare: douze ans, c'est l'âge auquel mourut le jumeau de Judith, Hamnet. 
La pièce voit la deuxième et dernière libération d'Ariel à l'âge de 24 ans répartis en deux moitiés, la première comme captif de Sycorax, la deuxième au service de Prospero. Eut-il été libre sous la souveraineté de la sorcière, que serait-il devenu d'autre que Caliban ? Où pouvons-nous l'imaginer après sa libération par Prospero, sinon au ciel ?

Si nous ne retenons que le sens de ces rapprochements, en oubliant les chiffres - j'avais prévenu qu'aucune causalité linéaire ne serait applicable ici - nous devons considérer que la pièce a pour objet la libération de Miranda-Judith de la tutelle de son père par son mariage et celle de la mort de son doublet : Ariel-Hamnet.

Qu'est donc cette île qui sera rendue à son désert à la fin de la pièce '? Nous nous sommes attachés à saisir le plus possible le sens de son peuplement, le temps du spectacle, mais peut-être n'avons-nous fait que le tour de ses côtes sans pénétrer plus avant dans sa signification en tant que scène. Elle est le lieu où règne Prospero. Un honnête nom bien italien. Entendons-le autrement : Pro-spero : "Pour ce que j'espère", ou mieux encore, "Pro-spear" : "Pour la lance", celle du nom de Shakespeare et du symbole qu'il fit figurer dans les armoiries (contestées) de son père, le bailli déchu de Stratford, substituant aux pacifiques colombes des armoiries de sa mère, Mary Arden, un oiseau de proie tenant une lance. Prospero - Antonio ${ }^{23}$, encore une histoire de trahison fraternelle, comme dans bien d'autres pièces, mais poussée à sa tension la plus haute dans Hamlet. Ici, la pomme de discorde, Gertrude n'a pas sa place. Son rôle est remplacé par une fille sans mère confiée à la garde vertueuse et veillant sur sa vertu, de son père.

La scène est donc en première approximation le lieu où les passions portent aux extrêmes. Leur spectacle ne suffit pourtant pas à nous révéler leur signification profonde, au delà des caractères qu'elles habitent et des situations compliquées qu'elles excellent à former. Pour rendre l" "intrigue humaine" - comme on dit la condition humaine - plus réelle, il faut avoir recours aux pouvoirs que peut s'octroyer le "représenteur". Montrer que sa magie n'est que la réalité de la réalité qui dépasse la fiction, non à titre d'exception, mais au contraire comme dévoilement de ce qui, encore et toujours, règne sur l'exceptionnel comme sur le quotidien, commande le cours des vies. Pour parvenir à cette pleine conscien$c e$, il faut alors que la scène s'anime, change, se transforme, passant de cet état obscur où elle nous apparaît d'abord sous le voile de la Tempête, à la clarté finale. "The island, écrit Northrop Frye, 
[...] is a place of confused identity in which a world of nothingness, symbolized by the tempest and the sea, separates from a world of regained identity..."24.

Si inhabitée qu'elle soit, nous avons vu que son désert est loin d'être un espace uniformément vacant. Tout au contraire, il suggère l'existence de présences menaçantes ou dangereuses, cachées ou mal localisables. Mais surtout, celui qui en est devenu le Maître s'abrite dans un lieu qu'il invitera ses invités à visiter à la fin : la grotte où son savoir se distille avant qu'il dispose de ses filtres pour fomenter ses actions. Une île dans l'île.

Non, ce n'est pas le cabinet de travail de Shaliespeare, c'est sa pensée. Là où il peut faire le vide, installer en lui cette scène déserte qui efface ce qui se rapporte à sa vie pour n'être plus ayant fait table rase - que le représenteur à qui nul n'a le droit de poser des questions sur les relations qui unissent le monde de sa création et le monde intérieur de celui qui crée. Un représenteur, ça représente. Quoi ? Ce n'est pas son affaire. A vous de le dire, qui en êtes le spectateur. Mais attention, ne prétendez pas le retenir dans votre grotte. Elle est trop petite, trop simple. Apprenez plutôt à tolérer le dissemblable, l'hétérogène, l'étrange et le merveilleux.

11 n'y a pas d'uniformité dans le spectacle qui se déroule sous nos yeux, l'action s'interrompant à tōut moment, immobilisant les acteurs pour réduire leurs actions à l'impuissance. $\mathrm{Ou}$ alors, voici ceux-ci, brutalement plongés dans un sommeil ${ }^{25}$ qui les retire momentanément du monde. Car on ne saurait dire absolument si Prospero se borne à suspendre la mise en œuvre de leurs désirs sur le monde de la scène, ou s'il n'exerce pas sur eux un droit de vie ou de mort, rouvrant, le cas échéant, les tombes à sa guise, quand tel est son bon plaisir, ou plus précisément, quand cela est nécessaire à la réalisation programmée de ses plans. 11 accroît le sentiment des personnages de baigner dans une réalité qui ne leur est pas familière en leur envoyant, grâce à ses aides, des visions. 11 trouble leur expérience antérieure en donnant à leurs sens des perceptions incongrues. $N$. Holland a raison de dire que $L a$ Tempête est une pièce sur les pièces ${ }^{26}$. En somme Shakespeare fait sentir aux acteurs - qui sont des hommes - qu'ils vivent sur scène d'une vie qui se déroule dans un autre monde dont il est seul à promulguer les lois.

Prospero revêt à l'occasion le manteau de sa royauté magicienne en attendant de pouvoir réendosser celui qui lui conférait 
l'autorité dans son duché. Mais il se joue de ceux qui lui envient cette parure en leur montrant que s'ils s'habillaient ainsi, loin de les grandir, ces habits ne feraient que faire apparaittre leur petitesse. Ce monde féérique dont il commande les allées et les venues et sur lequel il lâche tempêtes et arcs-en-ciel pour l'émerveillement de ceux qui assistent à ces prodiges, il ne faut pas l'opposer trop schématiquement au chaos qui menace à ses frontières. Les puissances infernales aussi doivent être gouvernées.

Destiny has to instrument

This lo'wer world and what is in it

Prospero peut nous apparaître parfois comme cet Hadès magicien et omniscient retenant quelque Proserpine. Il se défend de cette séquestration parce qu'il préfere donner sa capture à un époux que de la rendre à la propriété exclusive d'une Déméter qui bientôt verrait Sycorax renaitre de ses cendres dans cette nouvelle fonction. C'est ce que lui a appris le savoir des livres. Pas ceux qui sont dans les bibliothèques mais ceux qu'il a su acquérir dans le renoncement au pouvoir temporel. Ceux qui s'adressent à l'imagination, ceux qui nous apprennent l'étoffe de rêves dont nous sommes faits. $\mathrm{Si}$ la grotte est le refuge des livres, l'île est ce continent des rêves, protégée par la mer du sommeil.

Quand on règne sur un duché, c'est une erreur de croire que le pouvoir temporel peut se séparer du reste et se céder sans péril en conservant son autorité. Si vertueuses que soient les intentions paternelles, s'isoler avec sa fille pour l'éduquer et l'instruire ne met pas à l'abri de Caliban qui continue de faire entendre sa voix au milieu des sortilèges dont le savoir aura donné plus facilement la maitrise que celle de ses propres passions.

La nature peut aussi s'insulariser en l'homme, restant sourde aux appels à la transformation venus de l'action civilisatrice des hommes. Et quelle ironie déjà ! dans ce "brave new world" qui n'est si brave que d'appartenir, en fait, à l'ancien. Quel don de prophétie en effet !

Tous se sont accordés à reconnaître ce que Shakespeare dit ici : que la vraie vertu est dans la domination des impulsions, l'éloignement de la luxure et qu'il n'y a pas d'autre libération qui vaille en dehors de celle qui affranchit de l'esclavage des passions. L'exhortation morale est-elle néanmoins son affaire ? Non qu'il faille tenir pour négligeable cette méditation, mais elle est tellement étrangère à ce qui se dégage de son théâtre ! Ne pourrait-on y 
voir cependant la conclusion de son expérience `? Force est de dire que s'il était parvenu au but qu'il recommande d'atteindre nous aurions été privés de ses créations les plus prestigieuses et si c'est là vraiment sa conclusion, prenons acte alors qu'elle coïncide avec la cessation de sa création.

Non, la seule domination qui vaille est celle de cet espace, la scène, une île déserte, pour faire pousser sur cette nature sauvage les fruits de la passion théâtrale.

Cette Tempête dont nous disions qu'elle déchire le temps, qu'elle en suspend le cours pour déployer les figures démultipliées de la tri-unicité, fait dire à Prospero, à la fin :

And thence retire me to Milan, where

Every third thought shall be my grave

Et si à mon tour je vous demande pardon de vous avoir retenus trop longtemps, le crime dont je me serai rendu coupable est peut-être d'avoir trop voulu montrer ce qui est inutile au plaisir de la représentation.

André GREEN

Membre de la Société Psychanalytique de Paris

NOTES

1. "We split, we split" - "Farewell, my wife and children!" "Farewell brother !" - "We split, we split, we split!"

I, $1,1,59-60$.

2. Jean-Jacques Mayoux, Shakespeare, Aubier, p. 157.

3. André Green, Hamlet et Hamlet, Balland, 1982.

4. G. Wilson-Knight, The crown of life. The Shakespearian Superman, p. 206, Methueen.

5. Ella Sharpe, "From King Lear to The Tempest", in Collected Papers on Psychoanalysis, 1950, Hogarth Press.

6. Loc. cit. p. 225. «But poetry works to render fully objective the deepest ' $I$ ' intuition; as in Hamlet, himself so much the ' $\mathrm{I}$ ' of every spectator or reader. In his concentration on objective, especially natural, phenomona the poet expresses, as in a mirror common to all, his most intimate dreams and sorrows, while similarly in-reading subjective existence, the 'I' quality (no normal thought-currency gets the distinction) into the supposedly inanimate world.» 
7. Hallet Smith, Introduction à la Tempête, The Riverside Shakespeare, p. 1606, Houghton, Mifflin, Boston.

8. Hamlet et Hamlet, Balland, 1982, p. 259.

9. «Like all great poets, he has written regularly with strict respect for historical persons and events, well-worn mythology, thought-schemes not his own, sources of all sorts, sometimes an old play. This tendency marks not weakness but strength, and the resulting wholes become through that very self-loss constituent to his properly artistic realization; and it is precisely this "selfless artistic world" that The Tempest reduces to simplicity.» Loc. cit., p.225.

10. lbid., p.255. «This realization is, moreover, superpersonal and therefore universal. What is generally called a man's spiritual autobiography is accordingly less important than his artistic autobiography. Such an autobiography is The Tempest, isolating and intensifying the grand objectivity that characterizes the life-work it reduces to simplicity; for ever witnessing that the depths of personality blossom impersonally and that finest self-penetration is one with universal statement in the creative order where subject and object are felt as twin aspects of the more rounded, indescribable, whole.» romaine.

11. Pierre Grimal, Dictionnaire de la mythologie grecque et

12 André du Bouchet traduit librement: «Le capitaine, le pilote et moi» tandis qu'apparaissent au vers suivant le cannonier et le calfat. P. Leyris : «Le timonnier, le cannonier, le capitaine / Le gabier, le patron et moi». Calfat ou gabier? To swab, c'est bien nettoyer, essuyer, mais un swab c'est aussi un lourdaud, un propre à rien. Dans la marine, une épaulette.

13. Three glasses: trois heures.

14. Stephano, en argot napolitain signifierait "estomac", selon une suggestion de B. Croce, cf. Frank Kermode, Introduction à l'édition Arden.

15. «His [Prospero] spirits hear me.» (II,2,3-5) Caliban dit toutes les menaces que ceux-ci font peser sur lui jusqu'à le rendre fou. II,2,14. C'est ainsi qu'il voit d'abord en Trinculo l'un d'eux, avant de verser dans l'opinion contraire qui voit en lui un dieu.

16. Si Caliban vient bien de cannibale on peut alors comprendre la place que Shakespeare accorde aux pulsions orales dans $L a$ Tempête. Celles-ci sont traitées soit sur le mode de la recherche de la satisfaction directe - le vin - soit sous la forme sublimée, celle de l'avidité.

17. Dès leur première rencontre, le père de Miranda est attentif à tout ce qui peut prêter à une telle interprétation. Ferdinand dit trop vite qu'il est le meilleur, oublieux de l'existence de son père. I, 2, 431434. 
18. On remarquera la proximité sémantique entre Claribel et Miranda Un couple frère-sœur (Ferdinand-Claribel) est dissocié, un autre se forme unissant des époux (Ferdinand-Miranda).

19. N. Holland, The Shakespearian imagination, Indiana University Press, 1964.

20. Quand un joueur joue piètrement en procédant à des sacrifices compensés par autant de prises de l'adversaire faute de pouvoir construire une stratégie, le jargon des joueurs qualifie cette manière de jouer de "casser du bois".

21. Il n'est que de penser aux emplois contradictoires du mot esprit qui désigne des réalités opposées certes mais s'apparente beaucoup plus aux idées de la superstition et des croyances populaires, mélange de crainte et d'émerveillement qui permettent de dégager une vision "spiritualiste" de l'auteur. Il y a trop d'ironie pour qu'on y adhère ou qu'on pense qu'il souhaiterait que nous y adhérions.

22. Sa dernière fille se mariera cinq ans après $L a$ Tempête, à l'âge de 31 ans. Six semaines après, il signera son testament.

23. Antonio, un des prénoms les plus utilisés par Shakespeare : père noble (Les deux gentilshommes de Vérone), marchand prêt à garantir de sa chair un ami — un amant, diront certains - qui s'éloigne de lui (Le marchand de Venise), noble vieil oncle (Beaucoup de bruit pour rien), brave capitaine qui sauve un Sébastien (La nuit des Rois, situation exactement inverse de La Tempête) et enfin, frère usurpateur ici.

24. Northrop Frye, A natural perspective, Harcourt Brace Jovanovitch \& Columbia Universiry Press, 1965.

25. Miranda parait tentée de s'endormir, cédant seule à un sommeil naturel, durant le récit de son père qui réclame toute son attention. Etrangement le pouvoir de Prospero semble ici rencontrer sa limite, mais c'est peut-être que Miranda se sent moins concernée par un passé pour elle révolu qu'intéressée par l'avenir inconnu qui est devant elle et qu'elle attend sans pouvoir l'imaginer. Il veut l'intéresser à l'histoire, alors qu'elle a hâte de rêver ... au prince charmant qui viendra la reveiller.

26. N. Holland, loc. cit. 